\title{
Airglow dynamics observations by Mini-EUSO
}

\section{Pavol Bobik $^{* a}$, Ondrej Matija ${ }^{b}$, Marian Putis $^{a}$, Frantisek Koval $^{b}$, Michal Vrabel ${ }^{b}$, Simon Mackovjak ${ }^{a}$, Jan Genci $^{b}$, Kenji Shinozaki $^{a}$, Mario Bertaina $^{c}$, Francesco Fenu $^{c}$, for the JEM-EUSO Collaboration}

${ }^{a}$ Institute of Experimental Physics, Slovak Academy of Sciences (IEP SAS), Kosice, Slovakia

${ }^{b}$ Technical University Kosice (TUKE), Kosice, Slovakia

' Dipartimento di Fisica, Universita' di Torino, Torino, Italy

E-mail: bobik@saske.sk, ondrej.matija@student.tuke.sk,

putis@saske.sk, frantisek.koval.2@student.tuke.sk,

michal.vrabel@tuke.sk, mackovjak@saske.sk, jan.genci@tuke.sk,

kenjikry@gmail.com, bertaina@to.infn.it, francesco.fenu@gmail.com

Mini-EUSO ("UV atmosphere" in Russian Space Program) will observe Earth night side atmosphere from ISS in the UV band. The observed light consists mainly of light produced by airglow. We investigate the feasibility to observe airglow dynamics by Mini-EUSO detector. The investigation focuses on observability of two effects. One is the observability of magnetic storms in airglow light, where we expect decreasing light production in UV during storms. The other is the possibility to detect patterns created by atmospheric gravity waves induced by tsunami waves.

35th International Cosmic Ray Conference - ICRC2017

10-20 July, 2017

Bexco, Busan, Korea

${ }^{*}$ Speaker. 


\section{Introduction}

The main Mini-EUSO objective is to observe UV-light night emission $(300-400 \mathrm{~nm})$ from the Earth atmosphere. Such observation offers possibility to study wide range of scientific phenomena like Extreme Energy Cosmic Rays (EECR), atmospheric physics, strange quark matter and bioluminescence [1]. This article is focused on atmospheric physics specifically UV airglow and its dynamics. We expect that Mini-EUSO provides useful data which brings insight to understand geographical and time variation of airglow, effects of tsunami events and geomagnetic disturbances on upper atmosphere. The first prediction that atmospheric gravity waves (AGWs) might be detectable and used for tsunami warning system purpose was published by Peltier and Hines [2]. First observation by ground-based camera was published in [3]. Till now, the AGWs genereted by tsunami wave was not observed from the Earth's orbit. Mini-EUSO will observe all light sources which radiate in upward direction as reflected, such as, zodiacal and star light and direct ones such as, artificial light and bioluminescence. We expect that permanent sources (zodiacal and star light) will be constant and will have minimal effect on observation of airglow dynamics. Temporal sources like artificial light and bioluminescence will be excluded from our analysis. Mini-EUSO will record 400 thousands frames per second ( $2.5 \mu \mathrm{s}$ time resolution) using a matrix of $48 \times 48$ pixels (spatial resolution at ground of $\sim 6 \times 6 \mathrm{~km}^{2}$ ). However, only the frames which pass trigger detection will be stored with this high temporal resolution [4]. For observation of airglow Mini-EUSO will sum 16384 frames to one slow mode frame with integration time $40.96 \mathrm{~ms}$. Approximately 24 summed frames with Mini-EUSO observations will be stored every second.

\section{Observability of airglow dynamics by Mini-EUSO detector}

Mini-EUSO on ISS will observe the atmosphere under the station orbit between latitudes $-51^{\circ}$ and $51^{\circ}$. To cover this area, we estimated airglow production globally for set of grid points covering all globe. For each grid point we evaluate airglow production for more than two consecutive solar cycles. We call those grids as maps of airglow production.

We produced the maps of airglow production for the 25 years from the year 1970 till the year 1994. The airglow intensities were evaluated in AURIC model (Atmospheric Ultraviolet Radiance Integrated Code) [5] for empirical input parameters i.e. Ap indexes, F10.7 index and geomagnetic coordinates. Maps cover the globe with 10 degrees grid latitudinal and longitudinal step. Production was evaluated for night time when Sun is deep enough under the local horizon, which means when Sun's zenith angle is greater than $110^{\circ}$. The example of airglow light production map in wavelength range $300-500 \mathrm{~nm}$ for $5: 00$ UT on 9. January 1980 is presented in the figure 1 .

Figure 1 displays airglow light production on different geographical positions in the Earth night side. The maximum production in some regions is a few times higher than in the most dark regions. Airglow intensities were evaluated for one whole night in every month, i.e. for 12 nights per year at every point of grid. In summary 7200 maps were evaluated, 288 for every year (12 nights x 24 hours) of tested 25 years long period. Based on this example Mini-EUSO has potential to see geographical variability of airglow production during every orbit. However, visibility of geographical variation on orbit scale could be very strongly suppressed by clouds, 


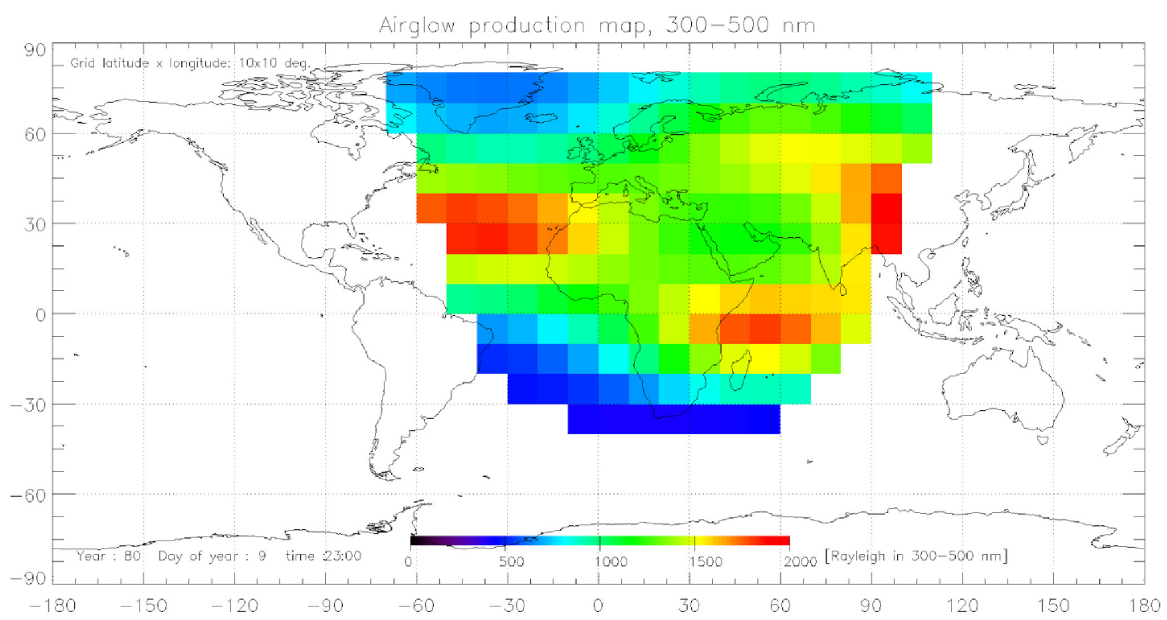

Figure 1: Airglow production at 9 January, 1980, 5 : $00 \mathrm{UT}$, in the wavelength range $300-500 \mathrm{~nm}$.

moonlight and other effects. Taking in account longer data collection of Mini-EUSO, arithmetic average of adjacent grid points could produce map comparable with model predictions.

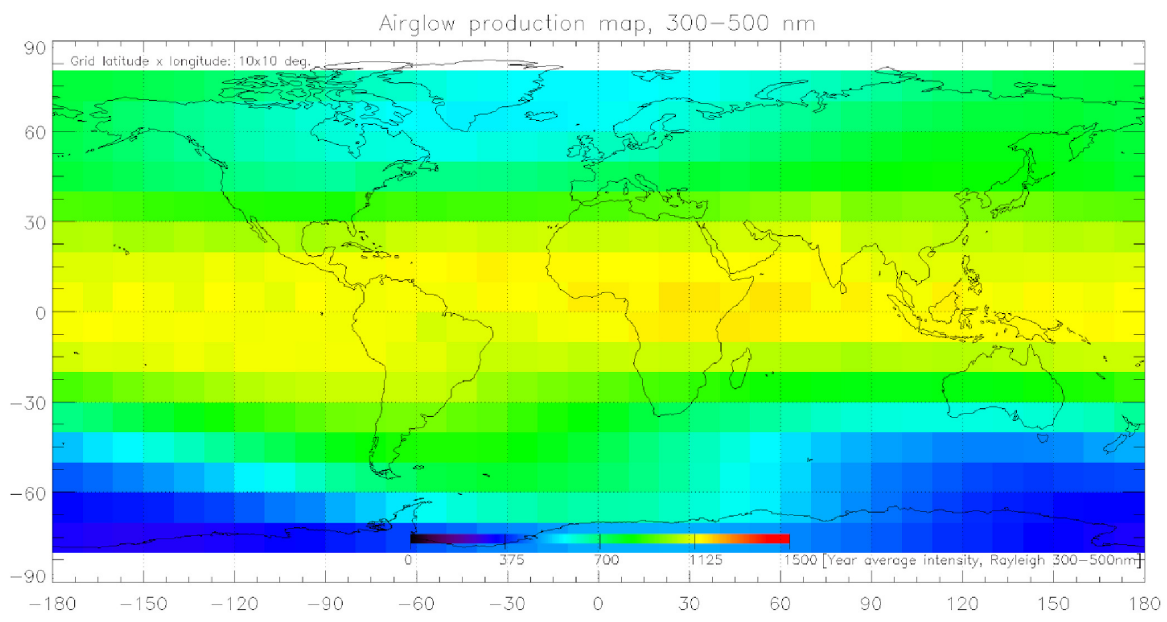

Figure 2: Annual average of airglow light for year 1980 (for details see text)

The map of average intensities in the year 1980 presented in figure 2 displays airglow production averaged from all night hours during the evaluated 12 nights. Figure illustrates geographical dependence of airglow light production. The average production in the wavelength range $300-500 \mathrm{~nm}$ is the highest in the equatorial regions.

In the figure 3 we show map of seasonal changes in the airglow light production. The averaged night intensities were used and difference between nights with maximal and minimal intensity values during the year is presented. The figure shows that seasonal variation is strongest in the middle latitudes of north hemisphere. The figure indicates that Mini-EUSO should see seasonal variation when comparing averaged map from months December and January with months as June and July. 


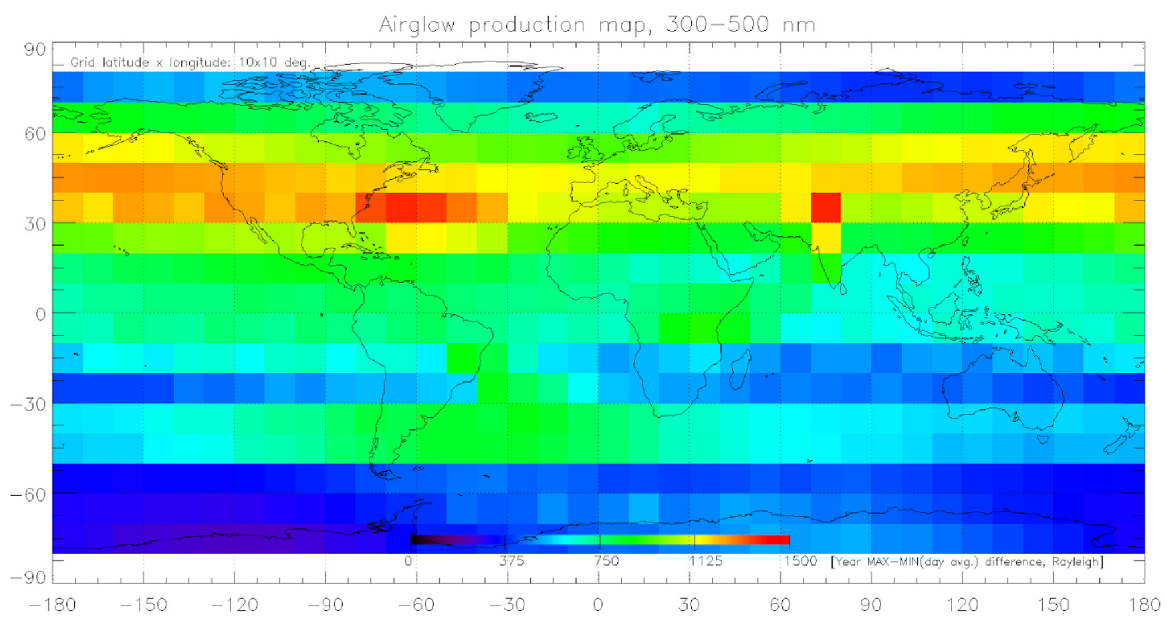

Figure 3: Seasonal variation of airglow light production in the year 1980.

\section{Observability of magnetic storms in airglow light}

The airglow light production depends along with other parameters also on geomagnetic conditions. We used simulations in AURIC model to estimate effect of geomagnetic storms to airglow production on global scale. Results from simulations give us image about scale of effect. Results indicate where effect could be observed by Mini-EUSO detector.

We produced two sets of maps for 25 year long period from 1970 to 1994 with identical time step and geographical grid as in previous section, both with constant geomagnetic and solar F10.7 indices over the whole tested period. First set has parameters for geomagnetically undisturbed periods $(A p=0)$, second for very disturbed periods $(A p=250)$. To exclude effect of solar activity expressed by $F 10.7$ index, constant value 140 for both calculations was used. Based on these two sets we calculate average relative deviation:

$$
\text { Average relative deviation }=\frac{1}{N} \sum_{i=1}^{N} \frac{\left(\left|I_{i A p=0}-I_{i A p=250}\right|\right)}{I_{i A p=0}}
$$

where I is intensity of airglow in Rayleigh units and $\mathrm{N}$ is number of calculated nights hours for given position. The result is presented in figure 4 .

The similar versions of figure 4 were prepared also for separate relative deviation intervals $(0-10 \%),(10-20 \%), \ldots(90,100 \%)$ and they are displayed in figure 5. Maps of average relative deviation in different intervals confirm that averaging didn't mix regions with very low and very high relative deviation. However some degree of mixing is present but in general, areas with highest relative deviation $(60-100 \%)$ are sufficiently separated from areas with low relative deviation $(0-60 \%)$.

ISS proceed one orbit during $\sim 92$ minutes. This mean $\sim 16$ orbit during one day. Each orbit is shifted to each other. Based on this we assume that during the mission time Mini-EUSO will produce global time dependent map of airglow radiation with relative sufficient spatial and time resolution. Based on such map we will be able to observe geomagnetic disturbances which produce fast decrease in airglow production. Night to night variation is less than $2 \%$ during the day and 


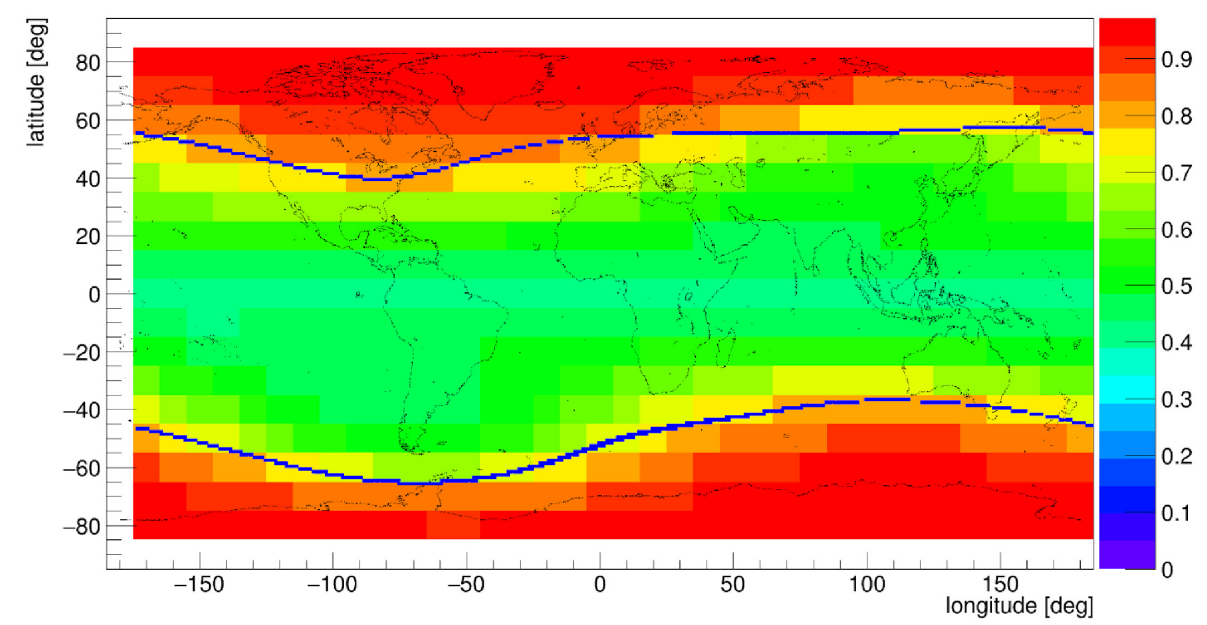

Figure 4: Average relative deviation between $A p=0$ and $A p=250$ during period between 1970 and 1994 . Borders of auroral oval at $K p=8$ are represented by blue line.

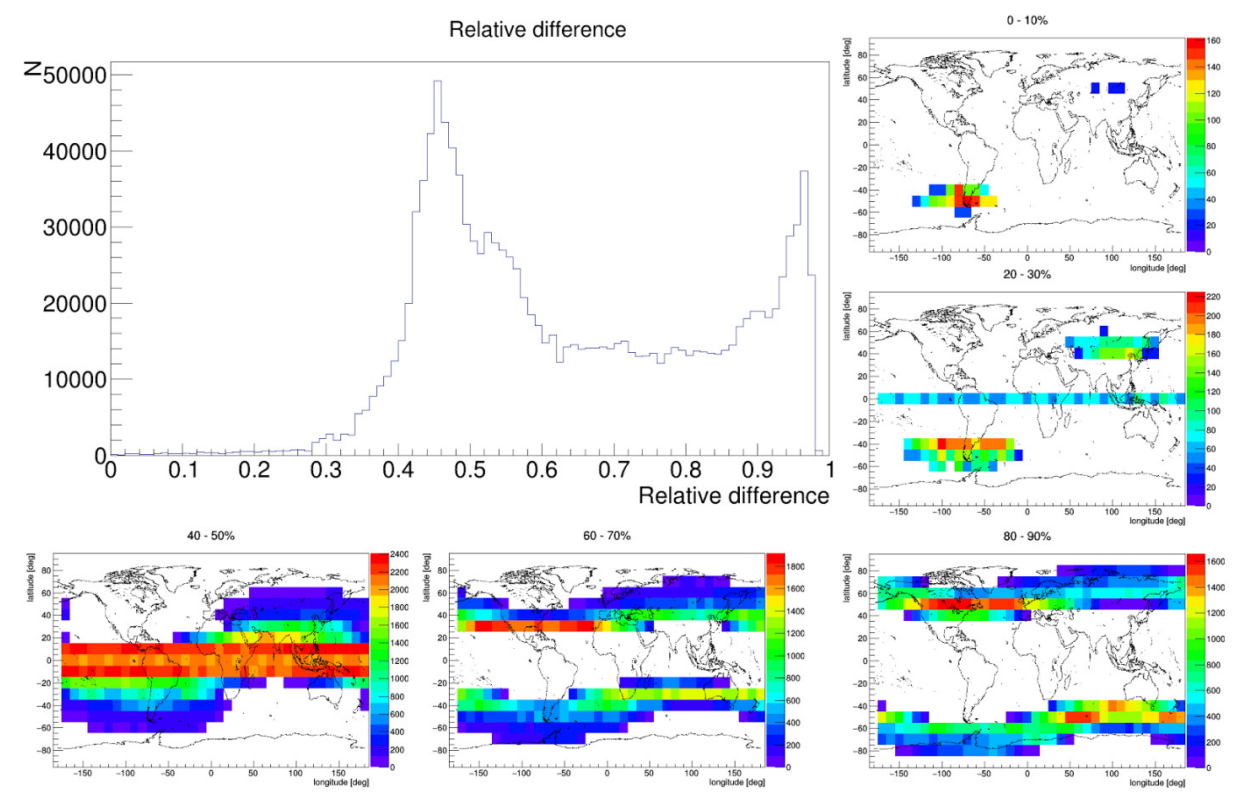

Figure 5: Airglow sensitivity to magnetospheric disturbances. Top left: Histogram of relative deviation ( $A p=0$ vs $A p=250)$ for all geographical grid positions. Top right and bottom: For given interval, histograms show number of occurrences for each position at map. Relative deviations are for period between 1970 and 1994.

is smooth, for this reason night to night variation will not hamper observation of geomagnetic disturbances.

Observation will be affected by clouds presence in the detector field of view. Clouds have high albedo and increase level of measured light significantly. For observation of geomagnetic effect only relative difference is important. For this reason only border between clear and cloudy conditions could produce noise for observation. Presence of such border could be identified because Mini-EUSO has resolution $48 \times 48$ pixels. Level of measured light will consecutive in- 
crease/decrease from one pixel to another if Mini-EUSO will fly over border of cloudy/clear sky conditions.

Based on this we assume that Mini-EUSO could produce sufficient data for observation of geomagnetic disturbances.

\section{Atmospheric gravity waves}

We were looking for optimal pattern recognition algorithm to identify and reconstruct a patterns of atmospheric gravity waves induced by tsunami waves in airglow light recorded by MiniEUSO. We simulated 16384 frames of $2.5 \mu \mathrm{s}$ each with UV light intensity equivalent to $500 \mathrm{ph} /\left(\mathrm{ns} \mathrm{sr} \mathrm{m} \mathrm{m}^{2}\right)$ to prepare one $40.96 \mathrm{~ms}$ frame of Mini-EUSO detector. Intensities at pixels were simulated with Poisson distribution. The generated wave in airglow light was summed with background.

Two pattern recognition methods were developed and tested, first one based on clustering algorithm, second based on Hough algorithm [6]. Both methods provide similar quality of recognition and reconstruction of wave parameters. We tested both method for three types of waves. Waves represented by damped sine wave, wave in form of wave package which had similar shape to that used in article [7] and wave from tsunami simulation package easyWave [8]. The methods precision was tested for different amplitudes of generated waves.

The example of test results is presented in table 1 where damped sine wave with $30^{\circ}$ angle to detector $X$ axis was used. Four amplitudes were tested and frames were processed by clustering method. Value 0.06 in table represent an amplitude of $6 \%$ of the signal due to the average UV background. Other values represent 12\%, 24\% and 48\% amplitudes. For every amplitude we generate one thousand slow mode frames. On every frame, pattern recognition algorithm selects wave top as cluster of interconnected pixels. Then wave position is reconstructed from cluster, as line $y=A+B x$ representing wave's top. The angle of wave to detectors field of view $X$ axis of coordinate system was evaluated from $B$ parameter and compared with simulated angle.The reconstructed angles and deviation from former value of angle has Gauss statistical distribution. We consider three values to describe statistical precision of reconstruction. The parameter $\gamma_{68}$ gives the information of which is the deviation angle which contains $68 \%$ of the results. In other words, in $68 \%$ percent cases the reconstruction was done with higher precision than value $\gamma_{68}$. Variable med is median of deviations distribution and $D H$ is bottom border of $1 / 3$ of deviations distributions from middle value.

Table results show that reconstruction has relatively high precision and $30^{\circ}$ angle could be in $68 \%$ cases reconstructed with better precision than $1.0^{\circ}$ for all tested amplitudes. The threshold value is clustering method parameter. The pixels for clustering are selected with values higher than this threshold. The precision of reconstruction is better for waves with higher amplitudes, however also wave with small $6 \%$ amplitude is recognized with high precision.

Following analysis tests clustering algorithm in more real conditions. We use ISS trajectory from NASA SSC locator [9]. For simulation of tsunami time traveling maps we used the easyWave package. We simulated tsunami map every 10 seconds for 24 hours long period. For this study we neglect effect of wave traveling to upper atmosphere and assume that maps of airglow production are identical with tsunami image. Amplitudes of AGW in airglow are scaled as $1 \%$ amplitude for $1 \mathrm{~cm}$ high wave on ocean surface. This assumption is supported by similarity of tsunami wave 
Table 1: Reconstructed angles in degree of damped sine waves with $30^{\circ}$ with the clustering method. The test was performed for 4 kinds of amplitude. $68 \%$ of reconstructed angles exist in $\sim$ med $\pm \gamma_{68}$.

\begin{tabular}{|c|c|c|c|c|c|c|c|c|c|}
\hline Thres. & \multicolumn{3}{|c|}{100.25} & \multicolumn{3}{c|}{103} & \multicolumn{3}{c|}{105} \\
\hline Ampl. & $\gamma_{68}$ & DH & med. & $\gamma_{68}$ & DH & med. & $\gamma_{68}$ & DH & med \\
\hline 0.06 & 0.2 & 29.78 & 29.97 & 0.21 & 29.78 & 29.98 & 0.95 & 27.79 & 29.66 \\
\hline 0.12 & 0.14 & 29.87 & 29.99 & 0.14 & 29.87 & 30.00 & 0.13 & 29.85 & 29.99 \\
\hline 0.24 & 0.1 & 29.89 & 29.99 & 0.10 & 29.91 & 30.01 & 0.09 & 29.90 & 29.98 \\
\hline 0.48 & 0.07 & 29.92 & 29.98 & 0.07 & 29.94 & 30.00 & 0.08 & 29.94 & 30.01 \\
\hline
\end{tabular}

shape and wave in airglow light in [7]. We used this approximate assumption to have basis for first analysis and estimation of tsunami wave visibility in Mini-EUSO observations. The simulations based on wave transport across the atmosphere and airglow production based on atomic oxygen volume changes is developed and will be presented in later article.

The Mini-EUSO FoV along the ISS trajectory was reconstructed with 10 seconds step. The figure 6 shows the tsunami wave from earthquake with moment magnitude scale $8.4 M_{W}$ in time $t=8800$ seconds after the start of tsunami propagation. The series of FoV with images seen by them is shown over the tsunami map. The clustering method recognizes wave in appropriate places as in $t=8900$ and 8960 seconds, even if amplitude of observed waves in FoV was only $\sim 6$ and $10 \%$.
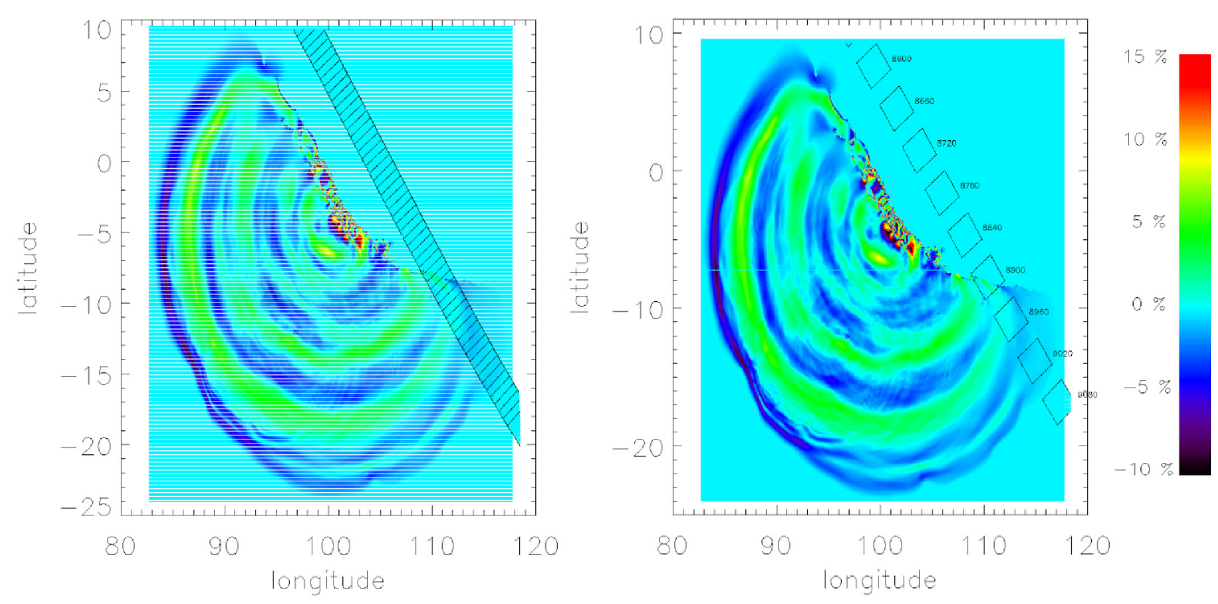

Figure 6: Example of Mini-EUSO flight over the tsunami. The Mini-EUSO FoV for the different positions on its orbit is indicated by the rectangles. The time after the tsunami occurence is shown next to each rectangle in the right panel.

\section{Conclusion}

Mini-EUSO at ISS will offer interesting possibility to observe UV airglow. ISS orbit cover broad range of geographical latitudes. This together with relatively long Mini-EUSO operation 
time ( hundreds of days) allow production of geographical and time dependent maps of UV upward radiation which include as most dynamic part airglow. These data bring insight to seasonal and geographical variation of airglow in ISS orbit area. Airglow depends also on geomagnetic field. Effect of changes in geomagnetic parameters during geomagnetic disturbances will be investigated in Mini-EUSO data. We expect decrease in airglow light level during such events. Mini-EUSO data offer interesting channel for exploration of geomagnetic disturbances.

The results from analysis preliminary indicate, that Mini-EUSO could register tsunami waves in observed airglow light during the cloudless observations/conditions. The influence of effects hampering a waves observations as clouds in FoV will be tested in further analysis.

\section{Acknowledgment}

We dedicate this proceeding to Yoshiya Kawasaki and Jacek Karczmarczyk, who passed away in 2016.

This work was partially supported by State Space Corporation ROSCOSMOS, as well as by Slovak Academy of Sciences MVTS JEM-EUSO, VEGA grant agency project 2/0132/17, ASI contract 2016-1-U.0 "MINI-EUSO" as well as by the government of Slovakia through an ESA contract under the PECS (Plan for European Cooperating States). ESA disclaimer: The view expressed herein can in no way be taken to reflect the official opinion of the European Space Agency.

\section{References}

[1] Valentina Scotti, Giuseppe Osteria for JEM-EUSO Collaboration, The Mini-EUSO telescope on the ISS, Nuclear Instruments and Methods in Physics Research A 845 (2017) 408-409.

[2] Peltier, W. R., and C. O. Hines, On the possible detection of tsunamis by a monitoring of the ionosphere, J. Geophys. Res. 81 (1976) 1995-2000.

[3] J. J. Makela, et al., Imaging and modeling the ionospheric airglow response over Hawaii to the tsunami generated by the Tohoku earthquake of 11 March 2011, GEOPHYSICAL RESEARCH LETTERS, 38 (2011) L00G02.

[4] G. Abdellaoui, S. Abe, A. Acheli, et al., Cosmic ray oriented performance studies for the JEM-EUSO first level trigger, Nuclear Inst. and Methods in Physics Research A (2017).

[5] D. J. Strickland et al., Atmospheric Ultraviolet Radiance Integrated Code (AURIC):theory, software architecture, inputs, and selected results, J. of Quantitative Spectroscopy and Radiative Transfer $\mathbf{6 2}$ (1999) 689-742.

[6] R. O. Duda and P. E. Hart, Use of the hough transformation to detect lines and curves in pictures, Commun. ACM 15 (1972) 11-15.

[7] M. P. Hickey et al., Atmospheric airglow fluctuations due to a tsunami-driven gravity wave disturbance, JGR 115 (2010) A06308.

[8] Babeyko, A. Y., easyWave available at: http://trac.gfz-potsdam.deleasywave.

[9] http://sscweb.gsfc.nasa.gov/cgi-bin/sscweb/Locator.cgi. 